\section{Whales found where whaling was}

The North Atlantic right whale (Eubalaena glacialis) is one of the world's rarest cetaceans, and little is known about its wintering or summering grounds, hampering conservation efforts. Now, researchers have documented signs of the whale in an area that was a whale-hunting ground in the late 1800s.

David Mellinger at Oregon State University in Newport and his colleagues carried out a year-long acoustic survey at five sites in and around the Cape Farewell Ground waters, an area about 500 kilometres east of southern Greenland. The team recorded more than 2,000 whale communication calls, mainly between July and November, suggesting that the area is still an important summer ground for the creatures.

The data will help to guide the relocation of shipping lanes and restrictions on vessel speed to prevent collisions with the animals.

Biol. Lett. doi:10.1098/

rsbl.2010.1191 (2011)

\section{ECOLOGY}

\section{Kill one species to save the rest}

The loss of one species from an ecosystem can have unpredictable - and on occasion catastrophic cascading effects. A modelling study suggests a strategy for rescuing a troubled ecosystem: selectively remove one or more additional species.

Sagar Sahasrabudhe and Adilson Motter of Northwestern University in Evanston, Illinois, showed that removing or partially suppressing one or more species in a food web at key time points after one member has become extinct saves other members of the web from the same fate. The duo used several model food webs, as well as two webs modelled with data derived from real ecosystems - the Chesapeake Bay off Maryland and Virginia, and the Coachella Valley in Southern California.

The idea - a controversial one that may not sit well with some conservationists - relies on the fact that ecosystem networks tend to shift to a different stable arrangement after losing members.

Nature Commun. doi:10.1038/

ncomms1163 (2011)

For a longer story on this research, see go.nature. com/2sckoo

\section{PHYSICS}

\section{Laser travels forwards and back}

One way to identify poisonous gases, or the vapours released by explosives, is to detect the effect of these molecules on laser light beamed through them. But practical detection devices should send and collect the laser beam from the same side of the gas cloud. Arthur Dogariu and his colleagues at Princeton University in New Jersey have taken a step towards this goal by demonstrating backwards lasing in air.

They used an ultraviolet 'pump' laser to break up oxygen molecules. The same laser then excited the molecular fragments into generating an infrared beam. The region in the air that the pump laser was focused on was about 100 times longer than it was wide. So half of the infrared light was emitted forwards, and the other half travelled backwards towards the source. The returning beam carried fingerprints of other molecules in the air.

Science 331, 442-445 (2011)

\section{ANIMAL BEHAVIOUR \\ Drummed into submission}

Paper wasps divide the work of the colony between different castes: workers build and defend the nest, whereas individuals destined to become queens lay eggs. Wasps do not inherit these

COMMUNITY CHOICE

The most viewed papers in science

\title{
Lights on for drug delivery
}

\section{- highly Read \\ on pubs.acs.org in Dec 2010}

A biocompatible gel that sheds its load when exposed to ultraviolet light might be used for controlled delivery of drugs and other molecules inside the body.

Weihong Tan at the University of Florida in Gainesville, Xiaoling Zhang at the Beijing Institute of Technology and their colleagues created this gel by first dissolving polymers decorated with DNA strands in water. They added DNA fragments that bind to the polymer-bound DNA, crosslinking the polymers to form a gel. These fragments also carry light-sensitive azobenzene molecules that, if hit by ultraviolet light, cause the crosslinks to break, releasing any trapped molecules. The gel unloaded a variety of test cargoes, including nanoparticles, an enzyme and the cancer drug doxorubicin, in a matter of minutes. Visible light restores the hydrogel's structure. Langmuir 27, 399-408 (2010)

roles, but are instead set on a particular developmental path during the larval stage. Researchers have discovered how this occurs in the genus Polistes: queens use their antennae to drum near to or on nest cells containing larvae to turn them into workers.

Sainath Suryanarayanan at the University of Wisconsin-Madison and his group used an electrical device to simulate this drumming on colonies that produce Polistes fuscatus wasps (pictured) destined to become queens. The wasps that emerged had the lean body type of workers. The link between the drumming - which for larger Polistes species is audible outside the nest - and geneexpression changes is not clear.

Curr. Biol. doi:10.1016/j. cub.2011.01.003 (2011)

\section{NEUROSCIENCE}

\section{Root of resilience under stress}

Some individuals react coolly to stressful events, whereas others slip into depression.
Work in mice suggests that chemical modifications to the DNA may explain the difference.

Shusaku Uchida and Yoshifumi Watanabe at Yamaguchi University in Japan and their colleagues subjected two genetically distinct strains of mice to chronic stress and then measured various proteins involved in neuronal growth and maintenance. The strain known to succumb to stress had lower than normal levels of a protein called GDNF in the brain's striatum. The resilient strain had higher amounts.

The team found that histones - proteins that package up DNA and regulate gene transcription - on a section of the Gdnf gene were modified differently between the two strains. This led to Gdnf repression in the susceptible mice and increased expression in the more resilient strain. Neuron 69, 359-372 (2011)

\section{ONATURE.COM}

For the latest research published by Naturevisit:

www.nature.com/latestresearch 